\title{
Virtual Reality (VR) Advertising Communication Design Based on 3D Wireless Active Visual Sensing
}

\author{
Yunshan Geng $\mathbb{D}^{1,2}$ \\ ${ }^{1}$ School of Art, Xi'an University of Architecture and Technology Huaqing College, Xi'an, Shaanxi 710055, China \\ ${ }^{2}$ Silliman University, Philippines 999005 \\ Correspondence should be addressed to Yunshan Geng; gengyunshan@su.edu.ph
}

Received 10 November 2021; Revised 13 January 2022; Accepted 17 January 2022; Published 7 February 2022

Academic Editor: Gengxin Sun

Copyright (C) 2022 Yunshan Geng. This is an open access article distributed under the Creative Commons Attribution License, which permits unrestricted use, distribution, and reproduction in any medium, provided the original work is properly cited.

\begin{abstract}
With the vigorous development of the advertising industry, advertising in the era of intelligent media fills all aspects of our lives, and more and more intelligent media are applied to the advertising industry. The current three-dimensional advertising and interactive advertising are too fragmented in form, with three-dimensional advertising having a strong sense of threedimensionality but lacking interactivity and interactive advertising having strong interactivity but lacking visual attraction. How to integrate 3D advertising and interactive advertising in order to make advertising in the future has greater development potential and market, whether in the visual or interactive experience can attract the target consumers becomes very necessary. It is found that VR technology has a wide range of application prospects, and intelligent media advertising mediated by VR technology follows the trend of advertising industry development. This thesis uses VR (virtual reality) technology as a medium to study the integration of $3 \mathrm{D}$ advertising and interactive advertising in the smart media era. In the second phase, the development direction of VR as an advertising medium in the era of intelligent media is identified. The research on the integration of 3D animated ads and interactive ads under VR technology is launched, and the research on 3D stereoscopic and interactive design of advertisements in the era of smart media is a reflection and exploration of the future language of advertising design. And through the comparison with traditional advertising, it is concluded that the aesthetic subject of VR advertising is "polycentric," the aesthetic object. Then, we analyze the theoretical roots of the formation of the aesthetic characteristics of VR advertising from three levels: media technology, communication theory, and audience psychology. Finally, we return to the general environment of visual culture and explore the deconstruction of visual culture by the aesthetic characteristics of VR advertising in three levels: "symbol collapse," "audience reshaping," and "immersive communication."
\end{abstract}

\section{Introduction}

The continuous development of advertising media, in the practical sense, can make the form of advertising more threedimensional and interactive; with the continuous change of advertising media, VR (virtual reality) technology has become the media of the intelligent media era; virtual reality (VR) technology is a new image technology that integrates threedimensional sense of picture and interactive forms, is an important direction for the future development of advertising, and is also one of the current hot topics of image research [1]. Since the emergence of advertising media, there have been different media in different times, from print media to radio media, from TV media to computer media, in the era of intelligent media with interactive functions of cell phone media, and finally developed to three-dimensional advertising and interactive advertising integration of VR media [2]. At present, VR advertising has a unique advantage; at the end of 2016, the famous international virtual advertising company, Airpush, released the world's first research report on the effectiveness of VR advertising. Based on objective data, the researchers quantified the effectiveness of VR advertising versus traditional advertising [3]. The report describes: "If VR is used as the technical support for the advertising effect, advertising will increase by 1.5 to 1.8 times, especially in terms of product memory, VR-based advertising as a new type of advertising message will increase the message memory effect by 5 times and advertising message sharing will increase by 2 times. As the cycle of media replacement becomes 
shorter and shorter, the advertising industry itself should adapt to the general trend and make changes to itself [4]."

It is of great relevance to provide rich information for advertisers to create VR advertisements, to analyze the existing technical bottlenecks and future development expectations for technology platforms, and to guide the correct perception of VR advertisements for advertising audiences [5]. Visual culture is an interdisciplinary and emerging term that involves many disciplines such as literature, art, and philosophy. The study of visual culture as a systematic theory began in the West in the 1980s, while visual culture as a research branch was really grafted into the field of communication after the 1990s [6]. After human beings left the traditional tribal society of "face-to-face communication" and "oral transmission," and after the birth of writing, the communication of information began to be done through media. The printing press realized the visualization of spoken expression, and the radio, television, and Internet after the printing press all made the enhancement of the "visualization" of information the ultimate goal of technological change [7]. The popularity of visual culture is in line with the pace of media technology, and because visual communication is inseparable from the role of media, visual culture research has become an important area of media research. At present, the number of consumers using traditional media is decreasing, and there is a lack of supply and demand between functional distribution and consumer demand [8]. According to the statistical analysis of relevant data, mobile terminals account for 38\%, computer terminals account for $35 \%$, TV media accounts for $16 \%$, and paper media only accounts for $11 \%$ of the forms in which consumers get advertisements, and people over 50 years old are bit traditional media consumers. In addition, the traditional media also lacks technical support and is not strong enough for development. The media is single, information is scarce, information is closed, and advertising requires technical processing [9]. However, in the era of growing intelligent media, on the one hand, advertising presents the characteristics of audience communication, dispersal communication, and infinite communication, and on the other hand, consumer needs are becoming more and more personalized, participation and interaction are becoming stronger, and the concept of experience is becoming more and more diversified [10]. Under the radical change of the external situation of advertising, many traditional advertisements still follow a single boring communication mode, and the development situation, theme setting, language mode, means of communication, skill innovation, situation layout, and other aspects do not follow the trend to keep up with the frontier of technological development, which leads to the advantages of traditional media decreasing compared with intelligent media [11]. One of the main reasons why traditional advertising is difficult to develop is that there is no key technology support and a lack of wisdom media technology-oriented advertising such as three-dimensional, interactive experience, ergonomics, and personalized services, which is out of touch with the times in terms of technological innovation [12].

Three-dimensional production technology is not the only way of film and television advertising production; in film and television advertising, we have to correctly play the computer's three-dimensional technology in order to effectively highlight its highlights, so that the production level of film and television advertising and the creation of the magnitude of greater improvement. In the computer three-dimensional technology performance level, will focus on the consideration of film and television advertising production supply and demand, for some live-action risk factor is large, it is difficult to take the content of the field, can consider using three-dimensional technology to produce. In short, in the production process of film and television advertising to rational use of threedimensional technology.Only by making appropriate use of the benefits of three-dimensional technologycan we give full play to the appeal of three-dimensional technology and make the explosive power of film and television advertisingenter a new stage. This paper takes advertising aesthetics as a research clue, analyzes the subject of advertising aesthetics, the object of advertising aesthetics, and the standard of advertising aesthetics, and seizes the core of advertising marketing from the audience's point of view, and the conclusions and proposed development strategies provide reference materials and case examples for advertisers to understand VR advertising and get involved in VR communication. In addition, the paper is also relevant in educating the advertising audience about the emerging VR advertising, and it provides a reference for the advertising audience to improve their media literacy. Therefore, the relevance of this paper exists at three levels: advertisers, technology platforms, and advertising audiences, and the conclusions of the study provide development strategies for the three main actors of advertising campaigns to understand VR advertising.

\section{Related Work}

VR advertising is the most emerging form of advertising nowadays, but few books have been written on this topic, and most of the relevant discussions are focused on journals and online platforms with high timeliness. VR online marketing advertising is a form of advertising in which audiences wear VR headsets to experience products or services online using cell phones or computers and other Internet terminal devices. Because of its convenience, large audience, and ease of data statistics, it has attracted the attention of many brands, and various kinds of online experiential advertising have also let audiences have a "VR addiction" [13]. A typical example of VR online marketing advertising is the new Mercedes-Benz "long wheelbase EClass" VR ad launched by Sohu and Mercedes-Benz, which has also become an innovative move in the new media industry and automotive advertising. The format of the ad is to jump into the "VR Panorama Ad" page through the Sohu page ad, a new E-Class sedan parked in front of a glass office building; the user can swipe the phone 360 degrees to "walk" around the car to observe; when the user clicked on the LED headlights, the car lights will automatically light up and pop up. When the user clicks on the LED headlights, the lights will automatically light up and a detail page introducing its performance will pop up [14]. In addition, users can also remotely experience the "autoparking" function and can also click "scene switch" to experience the steering wheel details and stereo sound effects inside the car. 
In order to ensure the advertising effect and user experience, the ad has impeccable details, and any component in the car can be felt by the users by clicking and popping up the details page. This VR live-action experience ad breaks away from the conventional film and TV commercial format and dares to break through the experiment, making the ad itself with a strong sense of immersion and technology [15]. At the same time, the contemporary and intelligent form of the ad coincides with the slogan of the Mercedes-Benz EClass long wheelbase car, "The wise, the great," making the ad a technology news at the same time and spreading the effect with half the effort. The ads are usually in the form of paid experiences [16]. The audience wears VR glasses with a gamepad, and the VR glasses are interconnected with the computer terminals. The content of the ads is mainly in the form of VR live-action games and live-action homes, aiming to sell VR equipment and promote VR technology. The audience can experience the latest technology through the many projects in this offline "VR experience"; the most common forms of VR games are VR surgery, VR brush, VR CS, etc., in addition to VR smart home, VR bike, VR live roller coaster, etc. Unlike gamers sitting in front of computers and TVs, VR games are set in an exceptionally realistic scenario, where the audience is stimulated by a combination of auditory, visual, tactile, and other senses, seeing objects and scenes beyond reality, so the audience will hide, run, and attack as in real games; they can also walk around freely in the kitchen of VR smart homes, open cabinets, and unscrew faucets [17].

The VR offline experiential advertising also defines this new technology as a "toy," penetrates into the audience with a game, and takes "live experience" as the biggest attraction for "attention economy" marketing [18]. The biggest highlight is the "attention economy" marketing. The advertisers have opened the door to a new world for their audiences and at the same time have profited from the new technology as a marketing tool and have made a significant contribution to the promotion and popularization of "VR for all." Although TV and online ads can easily restore or recreate scenes, VR goes beyond TV and the Internet in that it is no longer limited to visual perception but rather to deeper sensory stimulation, such as smell, touch, taste, and other stimuli [19]. Therefore, the "scene" and "experience" have become the pursuit of VR advertising, changing the traditional advertising pursuit of images, words, and symbols corresponding to the framework of the constraints, which lighten the unnecessary auxiliary factors, the "scene" and "experience" in the first place. At the same time, traditional advertising needs to abstract the core value elements of products or services for symbolic packaging, in order to achieve the correspondence between products and symbols, so as to create the symbolic qualities as the unique selling point of the brand [20]. Therefore, traditional advertisements need to use words, music, colors, and other elements to make comprehensive packaging, but VR advertising no longer needs such meaningless blind packaging, nor does it need brands to shape the spiritual value or symbolic connotation of products [21]. Instead, through the sensory stimulation brought by the "immersive and interactive" all-round scenes, audiences can experience the brand culture and stimulate the desire to buy.

\section{3D Visual Communication of VR Advertising}

3.1. MediaSmart Media Advertising Fusion. VR is immersive, and it allows consumers to be fully engaged in the real world virtualized by VR and can attract consumers in a short period of time, giving them a strong sense of immersion and enabling them to have an extremely realistic and authentic experience in contact with the virtual environment. It can be said that VR (virtual reality) technology combines the advantages of three-dimensional advertising and interactive advertising to demonstrate the functions and characteristics of the products. People can not only view the performance and appearance of the product through VR (virtual reality) devices but also experience the fun of the product firsthand. The VR medium is three-dimensional, and the three-dimensional images of the images can bring a great visual experience to the consumers. In the traditional media era, we capture content through the eyes. When people read advertisements, they will start to associate the things that newspapers, books, and other media make to describe, and consumers can form imagination in their minds after receiving the content of the advertisement. The textual description of the original appearance of things can be endless imagination, in this age of seeing is believing, and cannot let consumers really understand the appearance of goods and the use of VR three-dimensional technology. In order to allow consumers more intuitively see the appearance and even internal structure of goods, the intelligent media era of virtual reality advertising was born, as shown in Figure 1. The VR media has characteristics, advanced VR technology based on modern platforms public media products based on modern platforms, flexible media portfolio becomes a reality, and messages can be put into carefully prepared advertising content in each VR device at any time. So the result of the integration of 3D advertising and interactive advertising is not to simplify advertising but to give it more personalization and appeal. The VR media change consumer information contact behavior; in the age of smart media, the change of consumer information contact behavior is accompanied by changes in the media.

If the existing $3 \mathrm{D}$ advertising and interactive advertising can be integrated and gradually form a unified model, then it can provide consumers with "personalized" information and bring a fashion culture to the young consumer group, such as $3 \mathrm{D}$ images to attract consumers' attention and interactive advertising to follow up the satisfaction of today's trends. The interactive ads follow the satisfaction of today's trends.

In the era of intelligent media how to integrate threedimensional advertising and interactive advertising, the author found after research, first of all, from sensory integration to experience integration. Most of the current advertisements are in the visual aspect, which are brought by simple advertising design and special effects operation, and consumers can only stay in the sensory visual experience of advertising. With the continuous development and application of VR technology, the future immersion experience will be more realistic. Second, from one-way communication to interactive communication integration, despite the advantages of traditional media advertising in creativity and special effects, but cannot change the status quo of one-way communication of advertising, it is 


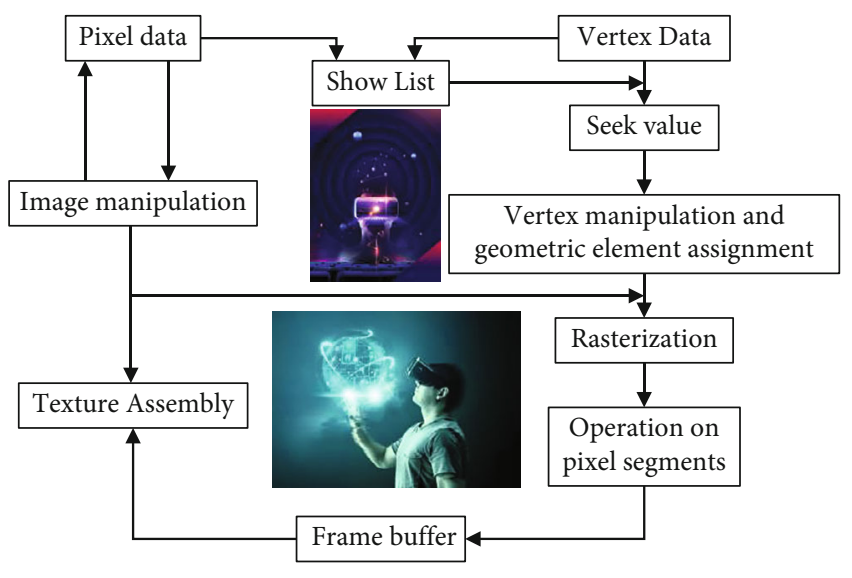

FIGURE 1: 3D VR technology and the evolution of 3D advertising.

difficult for consumers to interact with advertising. However, at this stage, too many experience technologies can only stay on some websites or touch screen and other devices.If you want to have an interactive experience in advertising films, you must use the interactive characteristics of VR media to integrate $3 \mathrm{D}$ advertising and interactive advertising. Advertising not only has a three-dimensional display technology, but also can interact with consumers, so that consumers are actively choose the need for advertising, enjoy the fun brought by advertising. In the process of using VR media technology to integrate threedimensional advertising and interactive advertising, advertisers will slowly reduce the use of traditional media. As VR technology becomes more and more mature, consumers are no longer just passive recipients.In other words, advertising is truly people-oriented. After the above analysis, I believe that when designing VR advertising, we need to focus on consumer immersion experience and interactive experience, using the characteristics of VR to design a real interactive environment to give consumers a better experience.

\subsection{Stimulate Consumption and Reduce Costs Based on VR} Technology. VR media change the behavior of consumer motivation.In the traditional media advertising era, most consumers will think that the media broadcast on the product has no problem.Often the advertising goods and brands equivalent to advertising companies, so when the consumers need to buy advertising goods, they will get information from the ads. But with the advent of the era of smart media, countless commodity ads broadcast on a variety of media has entered the era of ubiquitous advertising, especially the price reduction of electronic display products, the cost of advertising media is getting lower and lower, and the consumers began to change the past existing advertising viewing habits, from the beginning of passive acceptance of advertising to take the initiative to understand advertising and thus better able to search for the goods they need through the use of smart media VR. The use of intelligent media VR equipment forms of a sense of immersion in the real world. For advertisers, paying close attention to and even predicting the development trend of advertising and media make them willing to invest more time, money, and attention to advertising media. Because VR as a medium of advertising is a full-sensory immersive experience, with the advantages of both three-dimensional advertising and interactive advertising, allowing people to enter a fully immersive virtual reality realm, in this real "world," consumers can experience advertising more deeply and realistically, thus attracting a large number of consumers. Among the media used by young people, due to the rapid improvement of the performance and functions of electronic products, young people have more interest and dependence on the use of smart media, as shown in Figure 2. Nowadays, young advertising consumers are more attracted to $3 \mathrm{D}$ display media and media with interactive features, and advertisers are constantly trying to integrate the two technologies. As VR technology matures, VR-based media is becoming more available and, of course, more affordable. The use of VR technology not only allows consumers to view immersive $3 \mathrm{D}$ images but also allows consumers to independently select the ads they want, experience the interactivity of the product at home, and share their experiences and feelings in a timely manner, using consumer interactive reviews to advocate for the ad, making it better for promotion and distribution.

From the current technology, traditional media advertising has now formed a certain scale and experience, technology is also more mature, there is a fixed consumer base, and the popularity of VR devices is not high, so the study of VR technology is not very deep. The market use is not mature enough, because the development of three-dimensional advertising and interactive advertising has been more common.However, as for how to really put three-dimensional advertising and interactive advertising together, I think designers need to constantly upgrade their technical experience, think about how to improve the high level of consumer interaction and experience, and expand the influence of VR advertising on the market gravity, so that advertisers can create and design ads based on the characteristics of VR (virtual reality).By attracting consumers to promote VR devices and VR advertising, the integration will be faster and faster. Virtual reality advertising is popular in China and other countries as a new marketing model. In recent years, the 3D technology of VR has been widely used, using various techniques of virtual reality technology. For example, in the case of fierce competition in the real estate industry, the original form of real estate display has been difficult to meet the actual needs of consumers. Therefore, the real estate industry must grasp the trend of market development, making full use of the latest technology at present, breaking the original monolithic three-dimensional display on customers through the virtual reality sensors, and giving customers a strong three-dimensional sense of scene, which allows customers to walk in different house types and rooms and at the same time choose different decoration styles and furniture according to their preferences through the interactive function and to really feel the scene after decoration, which not only saves a lot of investment in model rooms and different decoration for the real estate industry but also greatly stimulates customers' desire to buy, which makes the sales of origin products grow rapidly (as shown in Figure 3). 


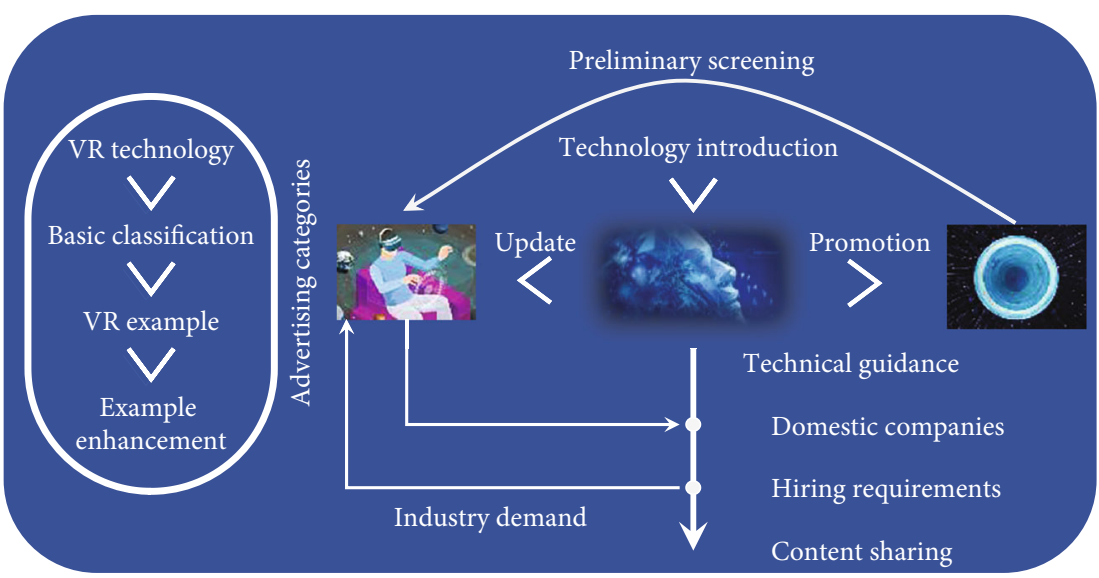

FIgURE 2: 3D VR media evolution process.

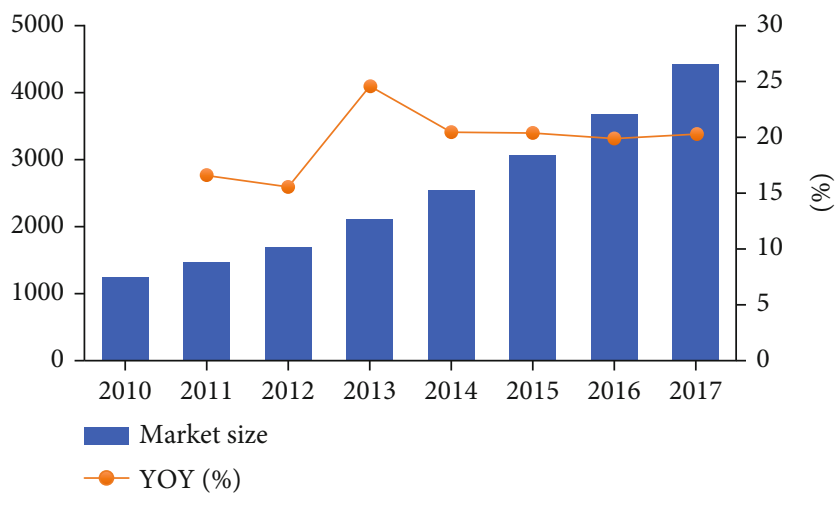

FIGURE 3: Global VR real estate market size forecast.

3.3. VR Display Advertising Design Practice. This advertisement is designed based on VR (virtual reality) technology as the medium. Through the analysis and research of the previous article, the author uses the design methods and design ideas summarized in the previous article as a practical guide; adopts the form of 3D immersive design interface, which can intuitively represent the real campus landscape and facilities; and adopts interactive design, which can interactively experience each landscape and facility and flexibly display each part of the campus to achieve the advertising. Because of its excellent immersion and immersion, the audience experiences the sensory stimulation even beyond the real, so the VR offline experience game is called "black technology" by users. The interactive design allows for interactive experience of each landscape and facility and flexible display of each part of the campus and achieves the advertising effect. This time, I did not choose ads with commercial nature but chose noncommercial ads with humanistic nature, hoping to promote the school with the help of VR media, so that more people can understand the charm of Ningjunyuan and integrate the culture of Ningjunyuan. In this advertisement, users can browse and watch the advertisement through computer or VR display device, and the typical buildings can be operated interactively. In order to reflect the authenticity of the display ads, the campus is filmed with an aerial camera for a bird's-eye view, and
3D max software is used to model the internal scenes of important buildings, especially the university's Union Building, and Pano2VR software is used to create hotspot interactions, so that users can use the virtual elevator to reach each floor and start consulting and answering questions with relevant university departments, or they can reach each branch of the university and become familiar with its specialties and advantages. That is to say, when a technology is applied to real life, its subsequent effects and consequences will not belong to the scope of human control; at the same time, people are addicted to the technological pleasure brought by technology on the one hand and are manipulated by this technological "pleasure" on the other hand.

The audience's sensory perception of information is roughly divided into the following five stages (Figure 4). The first stage is the initial stimulation stage of external physical information, and there is no great difference in the audience's perception of information at this stage; the second stage is the physiological filtering layer of the audience, i.e., the sensory stage. The third stage is the audience's psychological filtering layer; that is, the audience's own personalization and personality characteristics will be the second screening of information, and the role of emotion is the most important part of the link; the fourth stage is the audience's awareness of the product or brand. The fifth stage is the establishment of the cognitive profile, which has a certain chance; the audience's cognitive profile is not in the inevitable state that it will be established, but the confirmation is after the audience's perceived positioning of the brand or commodity awareness. It is a confirmation stage. In addition, the establishment of this stage will also provide feedback to the previous stages, and the desire for information generated by the establishment of the cognitive profile will have a rebound effect on the audience's mental and physical filters and continue to influence the audience's subsequent perception process.

In the eyes of "technological determinists," "technology is an independent force that develops according to its own logic, and although it arises from human needs, once it becomes a climate, it can easily become a wild horse out of human control." For example, before the technology of television was invented, there were no "TV people" and "container people." 


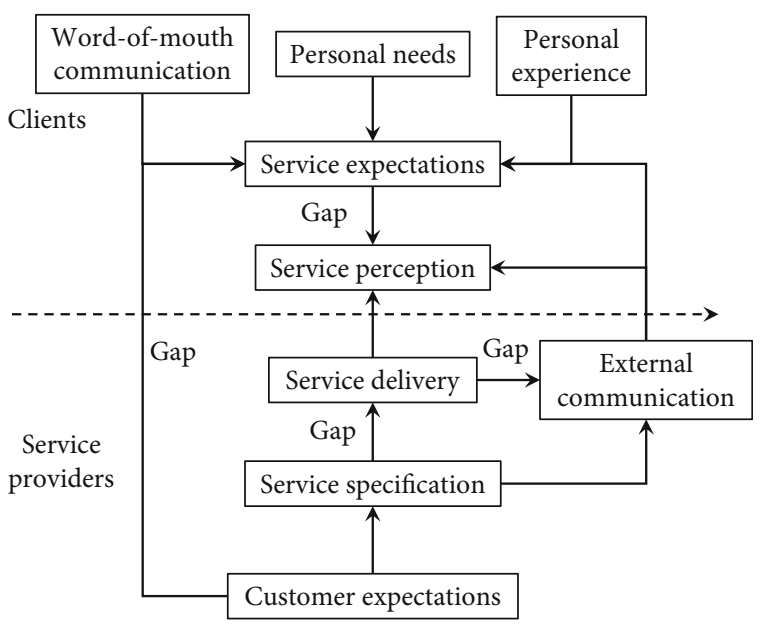

Figure 4: Consumer perception process model.

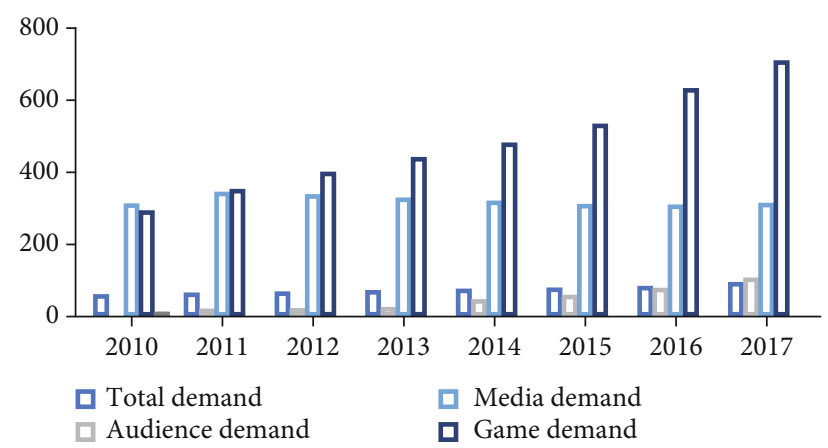

FIGURE 5: Demand generation in the dissemination process.

Before the technology of computer was popularized, there were no such terms as "mouse hand" and "Internet-addicted teenager." Once people reach this stage of "being controlled by technology," they become "puppets," that is, according to the logic of technology itself to change the human living environment and lifestyle and even to change the human value system. Therefore, VR advertising, as a hot new medium and technology, will also have a large or small impact on people's real life, and audiences should clearly understand that the "good" nature of technology and its "evil" nature exist together. The "change" of "technology changes life" is also neutral. VR advertising has opened a new chapter in people's understanding of the virtual world, but at the same time, it is also changing people's value identity, and the new trend in the aesthetic characteristics of advertising is the best reflection.

\section{The Development of Three-Dimensional Visual Psychology in Practice}

The "use and satisfaction" theory "views audience members as individuals with specific needs, and their media exposure activities as a process of using media based on specific needs and motivations, so that these needs can be satisfied." (1) From the concept, we can know that the theory involves three key words: demand, use, and satisfaction, and both "demand" and "satisfaction" are the psychological feelings

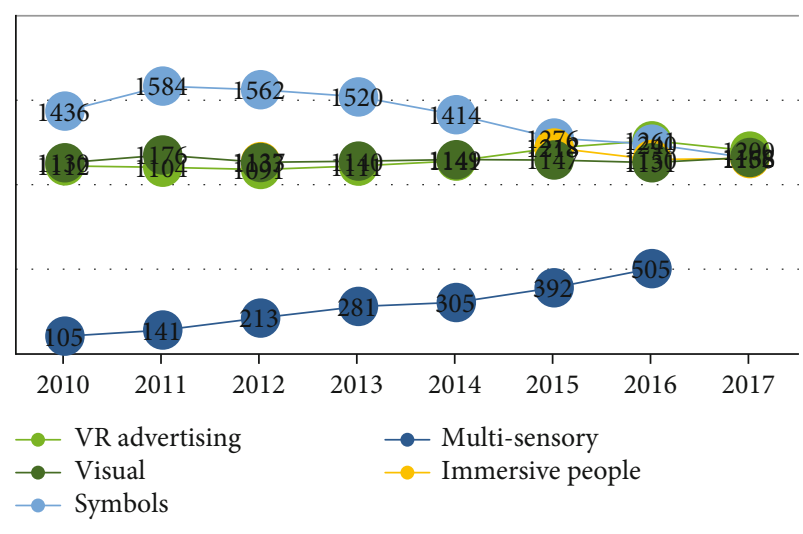

FIgURE 6: Sensory stimulation changes.

of the audience with autonomous and emotional characteristics, but "use" however is a dynamic process compared with the two and is a practical part of the construction of the audience's aesthetic psychological mechanism. After the construction of audience aesthetic mechanism based on audience sensory perception is completed, the subsequent behavior and psychology of the audience will also have a greater impact on the communication effect. The "use and satisfaction" communication process model shows the two main factors of demand generation (Figure 5), namely, social conditions and personal characteristics; after demand generation, the audience will choose the type of media based on media impressions and media contact possibilities and then engage in media contact. The whole process is bifurcated at the media contact stage, where the media that satisfies the audience's needs will in turn act on the media impression, and the audience will continue to seek other means of satisfaction if the media does not satisfy their needs.For advertising planners, VR creative will further unleash the imagination. Currently, there is only one way to combine VR and mobile marketing, so VR advertisers should work to innovate and inspire a richer and more diverse form of $\mathrm{VR}$ advertising.

From the above analysis, we can see that media impressions and media exposure possibilities are the key aspects of audience needs to be satisfied. In today's society, the richness of media forms gives audiences enough mobility and variety in their choices, so they pay more attention to their own physical and psychological pleasure of communication. This "selective exposure" gives audiences a great sense of pleasure and power, and they are more likely to follow their own media logic for media selection. This sensory perception also influences the image of VR media, and the positive image of the media attracts more and more attention from the audience. The development of VR advertising has received a great deal of attention in the past year, and it is very important for the audience to have a sense of satisfaction. The development of VR advertising has received a lot of attention in the past year, and people are full of curiosity and expectation for the new technology which is extremely human and interesting.

The derivation of new media and technologies represents a major advancement in human technology, and human beings have made remarkable achievements in the process of 


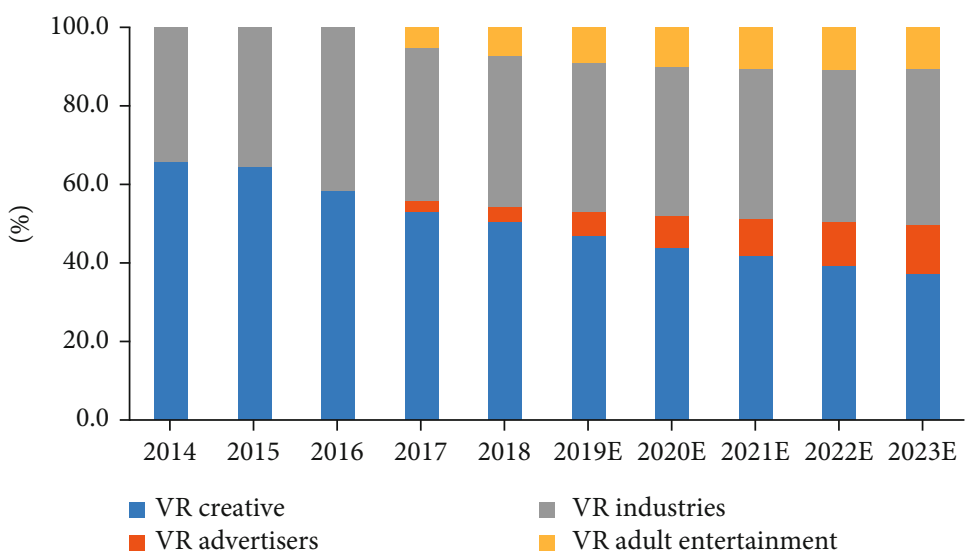

FIGURE 7: VR users' votes on "adult entertainment."

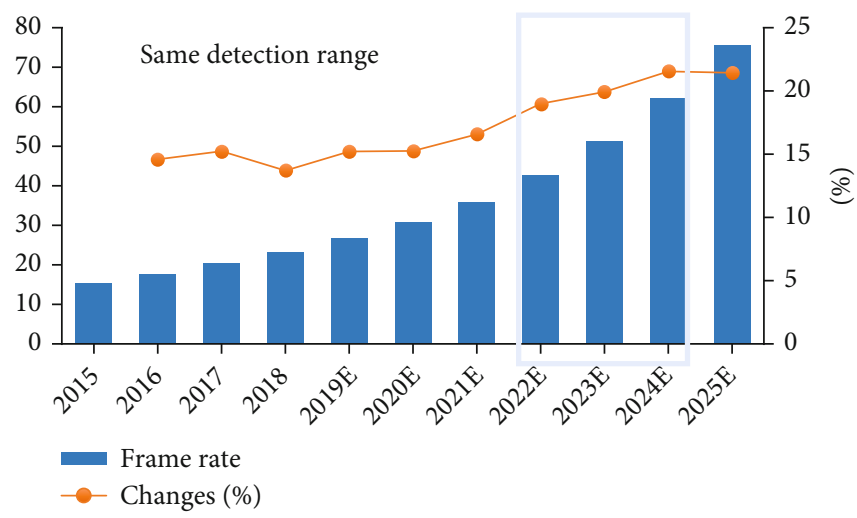

Figure 8: Frame rate variation of VR ads.

transforming the world. What we need to do is to take a high theoretical and global view of the transformation of real life by the new medium of VR advertising and to compare the properties of the new medium with those of the old medium, trying to understand the development of the new medium at its inception, as shown in Figure 6. The emergence of "immersive people" has reshaped the aesthetic psychology of advertising audiences; we are moving away from the "visual is king" approach. We are changing from a "visual" cognitive approach to a "multisensory-stimulating experience"-based exploration channel. All these changes prove that the change of media technology eventually leads to the renewal of the aesthetic characteristics of advertising.

First, VR advertisers should reject the temptation to strengthen their own ethical awareness. The Chinese VR user research report launches storm magic mirror in conjunction with the national advertising research institute on March 18, 2016.Now, Meng Consulting Agency has released the "Chinese VR user behavior research report," which report on China's VR users to conduct in-depth research; and in a comprehensive analysis of the current VR user behavior for the first time.The survey involved 15 provinces and cities across the country, and a total of 5,626 analysis samples were taken. The survey involved 5,626 samples taken from 15 provinces and cities for analysis. One of the survey data for the "top 10 VR industries of interest to users" showed that heavy users and light users voted $54.2 \%$ and $44.8 \%$, respectively, for "VR adult entertainment" (Figure 7). At present, more and more technology companies are investing in VR adult entertainment. Facing such audience and market situation, VR advertisers must strengthen their own ethical awareness, pay attention to their own moral shape, and not disrupt the whole VR market communication order because of immediate self-interest. Second, VR advertisers should strengthen their own strengths and improve their ability to innovate. In the face of the burgeoning VR market, advertisers should break the limits of the format and be willing to try more and fresher ways of communication. Fu Chuanzhi, general manager of Airwave mobile marketing at Omnicom Media Group, said, "For brand owners, VR devices will become the main link to consumers."

By focusing on the aesthetic characteristics of VR advertising as an object of study, the new aesthetic trends that emerge will have implications for both VR advertising research and the development of the advertising industry itself. The new characteristics of the aesthetic characteristics of advertising are inevitably disruptive to the existing visual culture: the role of "symbols" is gradually weakened. VR advertising has extremely demanding technical requirements, and any substandard 
equipment will have a direct impact on the effectiveness of VR advertising, so VR advertisers should improve their business capabilities, learn from the early start of VR technology and the development of mature foreign technology companies, and develop a series of technical development strategies combined with their own. First, technology platforms should improve their strengths and overcome technical barriers. Although VR advertising represents the most technological advertising available, there are still inevitable embarrassments in the technical equipment level. For example, the high price of VR devices, compared to smartphones, poses a barrier to audience reception, as shown in Figure 8; in addition, VR devices are prone to vertigo and discomfort due to their ultrarealistic panoramic views. How to break the price barrier and pave the way for the popularity of VR are the problems that VR technology platform needs to face.How to overcome the technical problemsand make the audience's experience reach an extremelyforgetful level is the proposition that the technology platform should be developed first.

First of all, VR advertising audience should strengthen their own media literacy. First, they should learn and master the ability to use new media means or equipment, which is the most basic ability that VR advertising audience should have; second, they should have the ability to read media information, that is, the reading and understanding of information; third, they should develop their own critical and questioning ability of media information, so that they will not be blinded by the complex information; fourth, they should strengthen the ability to collect, process, produce, and publish information. This is also the core ability that "immersion people" should have when they evolve to the stage of integration with the media. The shaping of the audience's "media literacy" is also an extremely important part of bridging the "digital divide." The audience should be aware of their "self-media" identity and strengthen their sense of responsibility and existence, so that they can sharpen their eyes in the face of temptation. Secondly, "opinion leaders" should also play their own role in guiding and cultivating the aesthetic ability of the audience. VR is currently the hottest medium of communication, and the trend has just emerged so that many companies and individuals cannot help but follow a trend, so the current communication market VR ads are numerous and mixed, not without some collective and individual exploit. The law has been broken, and some groups and individuals are taking advantage of it to disseminate information that is contrary to social mores. Mainstream media and opinion leaders should be aware of their own orientation and promote risk awareness to their advertising audiences. They should also be aware of their social responsibility to not promote VR ads that contain bad information in order to attract attention.

\section{Conclusion}

VR (virtual reality) has created a unique advertising logic, and its extension and experience in the field of advertising integration fully combine the immersion, three-dimensionality, and special services of VR (virtual reality) into the field of advertising, demonstrating its distinctive consumer attributes, which are released as a consumer attribute of commu- nication. But the continued maturity and development of technology will give rise to more advanced communication media, and each new media replacing the old media is a huge test for human beings. The change of media has its own "constant": we have to follow the old media, use the existing communication theory to verify the new media, and find the fit between communication theory research and social development in order to move forward in the turbulent stream of media change. "Whereas in the past interaction was the darling of a particular scenario, now consumption defines the properties of a certain product, a certain kind of release or liberation, and these dogmas are readily available whenever and wherever they are." In terms of broader content, it can be said that VR (virtual reality) or its equivalent is the perfect interplay of media people and crowds' tastes. The research on the integration of $3 \mathrm{D}$ animated ads and interactive ads under VR technology is launched, and the research on 3D stereoscopic and interactive design of advertisements in the era of smart media is a reflection and exploration of the future language of advertising design. In the era of smart media, the ads realized through the fusion of VR technology will become a new trend in the future development of advertising. The integration of $3 \mathrm{D}$ advertising and interactive advertising makes VR as a new medium in the age of intelligent media that has a new way out in the advertising industry, which can more easily and quickly replace the traditional media-based advertising methods, allowing consumers to have a more realistic and personalized advertising experience. In the future, mainstream media and opinion leaders should be aware of their own orientation and promote risk awareness to their advertising audiences.

\section{Data Availability}

The data used to support the findings of this study are available from the corresponding author upon request.

\section{Conflicts of Interest}

The authors declare that they have no known competing financial interests or personal relationships that could have appeared to influence the work reported in this paper.

\section{Acknowledgments}

This work was supported by the Shaanxi new liberal arts research and reform practice project:innovative research and practice of mixed teaching methods for art majors from the perspective of intelligent education.

\section{References}

[1] V. Bogicevic, S. Seo, J. A. Kandampully, S. Q. Liu, and N. A. Rudd, "Virtual reality presence as a preamble of tourism experience: the role of mental imagery," Tourism Management, vol. 74, pp. 55-64, 2019.

[2] K. Cowan, N. Spielmann, E. Horn, and C. Griffart, "Perception is reality... How digital retail environments influence brand perceptions through presence," Journal of Business Research, vol. 123, pp. 86-96, 2021. 
[3] S. Crawford-Holland, "Virtual healing: militarizing the psyche in virtual reality exposure therapy," Television \& New Media, vol. 20, no. 1, pp. 56-71, 2019.

[4] L.-E. Dubois and C. Gibbs, "Video game-induced tourism: a new frontier for destination marketers," Tourism Review, vol. 73, no. 2, pp. 186-198, 2018.

[5] M. F. Farah, Z. B. Ramadan, and D. H. Harb, "The examination of virtual reality at the intersection of consumer experience, shopping journey and physical retailing," Journal of Retailing and Consumer Services, vol. 48, pp. 136-143, 2019.

[6] L. de Gauquier, M. Brengman, K. Willems, and H. van Kerrebroeck, "Leveraging advertising to a higher dimension: experimental research on the impact of virtual reality on brand personality impressions," Virtual Reality, vol. 23, no. 3, pp. 235-253, 2019.

[7] S. H. Jeong, S. Kim, J. Y. Yum, and Y. Hwang, "Effects of virtual reality news on knowledge gain and news attitudes," International Journal of Mobile Communications, vol. 18, no. 3, pp. 300-313, 2020.

[8] J. Jung, J. Yu, Y. Seo, and E. Ko, "Consumer experiences of virtual reality: Insights from VR luxury brand fashion shows," Journal of Business Research, vol. 130, pp. 517-524, 2021.

[9] K. Kilic, M. G. Saygi, and S. O. Sezer, "Exact and heuristic methods for personalized display advertising in virtual reality platforms," Journal of Industrial \& Management Optimization, vol. 15, no. 2, pp. 833-854, 2019.

[10] K. W. Lau and P. Y. Lee, "Shopping in virtual reality: a study on consumers' shopping experience in a stereoscopic virtual reality," Virtual Reality, vol. 23, no. 3, pp. 255-268, 2019.

[11] Y.-J. Lee, W. Zhao, and H. Chen, "Consumer response to virtual CSR experiences," Journal of Current Issues and Research in Advertising, vol. 42, no. 1, pp. 102-122, 2021.

[12] W. H. Lo and K. L. B. Cheng, "Does virtual reality attract visitors? The mediating effect of presence on consumer response in virtual reality tourism advertising," Information Technology \& Tourism, vol. 22, no. 4, pp. 537-562, 2020.

[13] A. Marasco, P. Buonincontri, M. van Niekerk, M. Orlowski, and F. Okumus, "Exploring the role of next-generation virtual technologies in destination marketing," Journal of Destination Marketing and Management, vol. 9, pp. 138-148, 2018.

[14] S. Okolo, "Can virtual reality improve the health of older sports fans," IEEE Potentials, vol. 38, no. 3, pp. 17-19, 2019.

[15] J. Park, J. Choi, and C. Rhee, "Futuristic VR image presentation technique for better mobile commerce effectiveness," Virtual Reality, vol. 25, no. 2, pp. 341-356, 2021.

[16] G. Pizzi, V. Vannucci, and G. Aiello, "Branding in the time of virtual reality: are virtual store brand perceptions real?," Journal of Business Research, vol. 119, pp. 502-510, 2020.

[17] N. Spielmann and U. R. Orth, "Can advertisers overcome consumer qualms with virtual reality?," Journal of Advertising Research, vol. 61, no. 2, pp. 147-163, 2021.

[18] C.-Y. Ung, M. Menozzi, C. Hartmann, and M. Siegrist, "Innovations in consumer research: the virtual food buffet," Food Quality and Preference, vol. 63, pp. 12-17, 2018.

[19] P. Vishwakarma, S. Mukherjee, and B. K. Datta, "Antecedents of adoption of virtual reality in experiencing destination: a study on the indian consumers," Tourism Recreation Research, vol. 45 , no. 1 , pp. $42-56,2020$.
[20] R. Yung, C. Khoo-Lattimore, and L. E. Potter, "Virtual reality and tourism marketing: conceptualizing a framework on presence, emotion, and intention," Current Issues in Tourism, vol. 24, no. 11, pp. 1505-1525, 2021.

[21] R. Yung, C. Khoo-Lattimore, G. Prayag, and E. Surovaya, "Around the world in less than a day: virtual reality, destination image and perceived destination choice risk in family tourism," Tourism Recreation Research, vol. 46, no. 1, pp. 3$18,2021$. 